\title{
ON THE NORMALITY OF HIGGINS COMMUTATORS
}

\author{
ALAN S. CIGOLI, JAMES R. A. GRAY, AND TIM VAN DER LINDEN
}

\begin{abstract}
In a semi-abelian context, we study the condition (NH) asking that Higgins commutators of normal subobjects are normal subobjects. We provide examples of categories that do or do not satisfy this property. We focus on the relationship with the Smith is Huq condition $(\mathrm{SH})$ and characterise those semi-abelian categories in which both $(\mathrm{NH})$ and $(\mathrm{SH})$ hold in terms of reflection and preservation properties of the change of base functors of the fibration of points.
\end{abstract}

\section{INTRODUCTION}

The recent works [27, 19] explain that the universal-algebraic commutator defined by Higgins in the context of varieties of $\Omega$-groups [21] can be defined in an arbitrary semi-abelian category 24. In contrast with the commutator introduced by Huq 22 —already in a setting essentially equivalent to semi-abelian - which is defined for a pair of subobjects $K, L \leqslant X$ as a normal subobject $[K, L]_{X} \triangleleft X$, a priori the Higgins commutator $[K, L] \leqslant X$ is in general just a subobject of $X$, even when $K$ and $L$ are normal subobjects of $X$. In fact the two are closely related, as the former is always the normal closure of the latter.

Since, in general, Huq and Higgins commutators do not coincide, their eventual coincidence, for normal subobjects, becomes a property that a semi-abelian category may or may not satisfy. This condition, which we will denote by $(\mathrm{NH})$, was introduced by the first author in his Ph.D. thesis [15]. In this article we study the condition $(\mathrm{NH})$ as well as its relation with other categorical conditions. In addition we give examples, counterexamples and equivalent characterisations.

In Section 1 we recall the definitions of the Huq and the Higgins commutator and some of their basic properties. In Section 2 we explain that (NH) holds if and only if, for any pair of (protosplit) normal subobjects of an object, these two commutators coincide (Theorem 2.8). It is well known that for a group $G$, the commutator $[G, G]$ is a characteristic subgroup of $G$. In Section 3 we show that this can be proved in a semi-abelian category satisfying $(\mathrm{NH})$, if the definition of characteristic subobject from [16] is used. In Section 4 we recall from the first author's Ph.D. thesis [15] that any category of interest in the sense of [31] satisfies $(\mathrm{NH})$ and we give a first example of a semi-abelian category which does not. In Section 5 we compare $(\mathrm{NH})$ with the Smith is Huq condition (SH) considered in [28], and show that the two are independent from each other. In Section 6 we characterise those categories which satisfy both $(\mathrm{NH})$ and $(\mathrm{SH})$ in terms of the fibration of points. In particular, Theorem 6.5 tells us that $(\mathrm{SH})+(\mathrm{NH})$ is equivalent to the condition that for any $f: W \rightarrow Z$ in $\mathscr{C}$, the change of base functor $f^{*}: \mathrm{Pt}_{Z}(\mathscr{C}) \rightarrow \mathrm{Pt}_{W}(\mathscr{C})$ of the

2010 Mathematics Subject Classification. 08C05, 17A99, 18G50, 20F12.

Key words and phrases. Commutator, semi-abelian category, fibration of points.

The first author's research was partially supported by FSE, Regione Lombardia. He would like to thank the Institut de Recherche en Mathématique et Physique (IRMP) for its kind hospitality during his stay in Louvain-la-Neuve. The third author is a Research Associate of the Fonds de la Recherche Scientifique-FNRS. He would like to thank the University of South Africa for its kind hospitality during his stay in Johannesburg. 
fibration of points preserves Huq commutators of pairs of normal subobjects. In fact, it suffices to have this condition for $W=0$, so $(\mathrm{SH})+(\mathrm{NH})$ holds if and only if the kernel functors $\mathrm{Ker}: \operatorname{Pt}_{Z}(\mathscr{C}) \rightarrow \mathscr{C}$ preserve Huq commutators of pairs of normal subobjects.

\section{Preliminaries}

Throughout this paper we assume that $\mathscr{C}$ is a semi-abelian category [24, 2].

Definition 1.1. 22] A pair of morphisms $f: A \rightarrow C$ and $g: B \rightarrow C$ is said to commute or cooperate if there exists a (necessarily unique) morphism $\varphi$ making the diagram

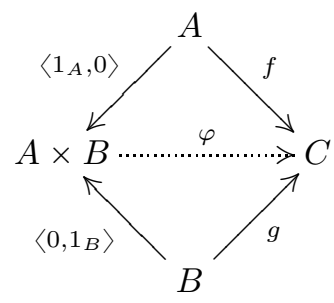

commute.

In this setting it can be seen that two morphisms commute if and only if their regular images commute (see for instance [2]). For this reason we will only define the Huq commutator for subobjects.

Definition 1.2. 22] For a pair of subobjects $k: K \longmapsto X$ and $l: L \longmapsto X$ of $X$ in $\mathscr{C}$, the Huq commutator is the smallest normal subobject $[K, L]_{X} \triangleleft X$ such that the images of $k$ and $l$ commute in the quotient $X /[K, L]_{X}$.

In this context [7, 2] it can be shown that Huq commutator of $k$ and $l$ always exists and can be constructed as the kernel of $q$ in the diagram

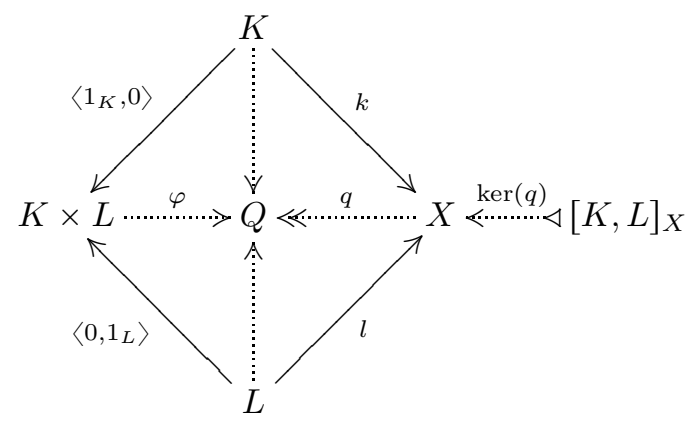

where $Q$ is the colimit of the square of solid arrows, or equivalently as the kernel of $q$ in the diagram

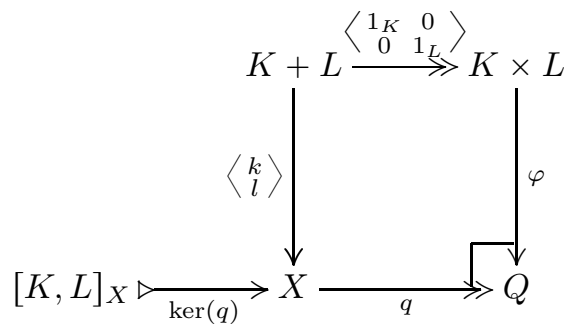

in which the right hand square is a pushout. 
Definition 1.3. 27, 19] For a pair of subobjects $k: K \longmapsto X$ and $l: L \longmapsto X$ of an object $X$ in $\mathscr{C}$, the Higgins commutator of $K$ and $L$ is the subobject $[K, L] \leqslant X$ constructed as in diagram

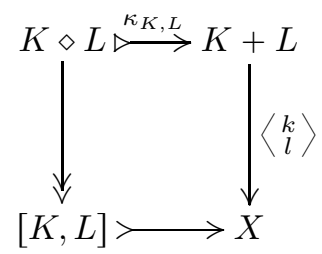

where $\kappa_{K, L}$ is the kernel of $\left\langle\begin{array}{cc}1_{K} & 0 \\ 0 & 1_{L}\end{array}\right\rangle: K+L \rightarrow K \times L$.

The object $K \diamond L$ is the co-smash product [14, 27, 20, of $K$ and $L$ and the Higgins commutator is its regular image under the morphism $\left\langle\begin{array}{l}k \\ l\end{array}\right\rangle \circ \kappa_{K, L}$. Note that the normal subobject $(K \diamond L) \triangleleft(K+L)$ can also be seen as the Huq commutator of the coproduct inclusions $\iota_{K}: K \rightarrow K+L$ and $\iota_{L}: L \rightarrow K+L$.

Examples 1.4. In the category Gp of groups, $K \diamond L$ is generated by words $k l k^{-1} l^{-1}$ as a normal subgroup of $K+L$, so that $[K, L]$ is the usual commutator of $K$ and $L$ in $X$. In the category CRng of (non-unitary) commutative rings, $[K, L]=K L$.

We recall:

Lemma 1.5. Consider $K, L \leqslant X \leqslant Y$. Then the Higgins commutator of $K$ and $L$, computed in $X$, coincides with the Higgins commutator of $K$ and $L$, computed in $Y$.

Proof. This follows from uniqueness of regular images.

Lemma 1.6. [27, Proposition 5.7] For $K, L \leqslant X$, the Huq commutator $[K, L]_{X}$ is the normal closure in $X$ of the Higgins commutator $[K, L]$.

Proof. Let $k: K \longmapsto X$ and $l: L \longmapsto X$ be subobjects of $X$. Consider the diagram

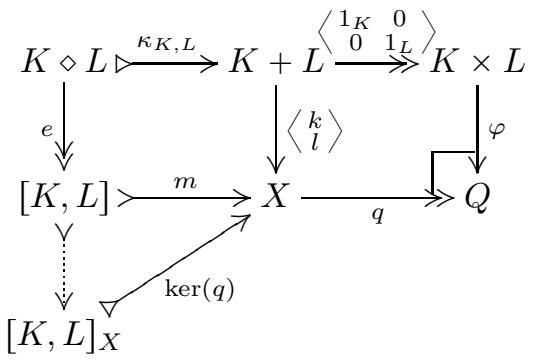

where $m$ is the image of the morphism $\left\langle\begin{array}{l}k \\ l\end{array}\right\rangle$. Since $\left\langle\begin{array}{cc}1_{K} & 0 \\ 0 & 1_{L}\end{array}\right\rangle$ is the cokernel of $\kappa_{K, L}$ and the square on the right is a pushout, it follows that $q$ is the cokernel of $m \circ e$. Since $e$ is an epimorphism, $q$ is also the cokernel of $m$. It immediately follows that the Huq commutator $[K, L]_{X}$ is the normal closure of $[K, L]$ in $X$.

Since in a semi-abelian category the regular image of a normal subobject is normal, if $\left\langle\begin{array}{l}k \\ l\end{array}\right\rangle$ is a regular epimorphism (i.e. when $X=K \vee L$ ), then the two commutators coincide.

As a consequence of Theorem 4.4, we will see that, for normal subobjects, the two commutators coincide in every category of interest in the sense of G. Orzech [31, such as Gp or the category Rng of (non-unitary) rings and $R$-Lie of Lie algebras over a fixed ring $R$. However, Examples 4.5 and 5.4 show that for an arbitrary semi-abelian category, even those which are closely related to categories of interest, the two commutators need not coincide for abitrary pairs of normal subobjects.

The Higgins commutator can also be used to characterise normal monomorphisms. 
Lemma 1.7. [27, Theorem 6.3] A subobject $K \leqslant X$ is normal in $X$ if and only if $[K, X] \leqslant K$.

In fact, a more general version of this result holds: Lemma 4.9 in [19].

\section{The CONDition (NH)}

In general, as explained above, Huq and Higgins commutators need not coincide; in other words, Higgins commutators need not be normal. In some categories, though, the Huq commutator of a pair of normal monomorphisms will always coincide with its Higgins commutator. In this section we focus on equivalent formulations of this condition.

Definition 2.1. A semi-abelian category satisfies the condition Normality of Higgins commutators (NH) when, for each pair of normal subobjects $K, L \triangleleft X$, the Higgins commutator $[K, L] \leqslant X$ is normal in $X$.

It is easy to check that the category of groups satisfies (NH). By contrast, the following example shows that, for arbitrary (non-normal) subgroups $K$ and $L$ of $X$, the commutator $[K, L]$ need not be a normal subgroup of $X$.

Example 2.2. 15, Example 5.3.9] Let $A_{5}$ be the simple group of even permutations of order five. For the two subgroups $K=\langle(12)(34)\rangle$ and $L=\langle(12)(45)\rangle$, we have $[K, L]=\langle(345)\rangle \neq[K, L]_{A_{5}}=A_{5}$ its normalisation.

Under (NH), Higgins commutators and Huq commutators of normal subobjects coincide.

Lemma 2.3. In a semi-abelian category with $(\mathrm{NH})$,

$$
K, L \triangleleft X \quad \Rightarrow \quad[K, L]_{X}=[K, L] .
$$

Proof. By Lemma 1.6 the Huq commutator $[K, L]_{X}$ is the normal closure of $[K, L]$ in $X$. Hence if $[K, L]$ is already normal in $X$, then the two commutators will coincide.

Lemma 2.4. In a semi-abelian category with (NH), if $K, L \leqslant X \leqslant Y$ and $K$, $L \triangleleft Y$, then

$$
[K, L]_{X}=[K, L]_{Y}
$$

Proof. If $\mathscr{C}$ satisfies $(\mathrm{NH})$, then it follows from Lemma 2.3 that both commutators $[K, L]_{X}$ and $[K, L]_{Y}$ coincide with the Higgins commutator $[K, L]$, which is independent of the object in which it is computed by Lemma 1.5 .

Lemma 2.5. Given $K \triangleleft X \triangleleft Y$, consider the induced diagram

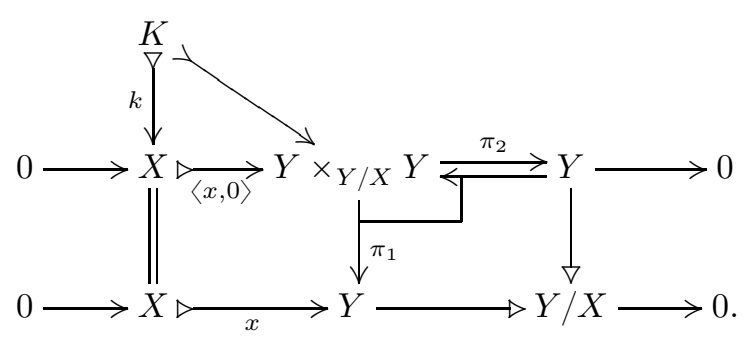

If $K \triangleleft Y$ then $K \triangleleft\left(Y \times_{Y / X} Y\right)$. 
Proof. Since $K \triangleleft Y$, the right hand side pullback square decomposes into a composite of pullbacks:

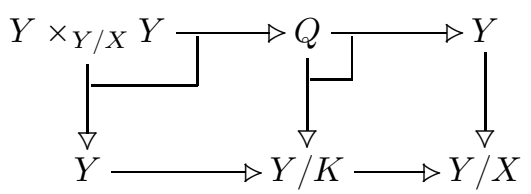

It is clear that $K$ is the kernel of $Y \times_{Y / X} Y \rightarrow Q$.

Recall the well known fact:

Lemma 2.6. Consider a split extension as in bottom row of the diagram

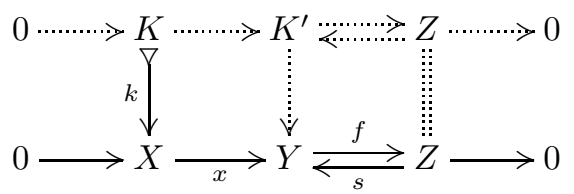

such that $x \circ k$ is normal. Then this split extension lifts along $k: K \rightarrow X$ to yield a normal monomorphism of split extensions.

Proof. The needed lifting is obtained via the pullback of split extensions in the diagram

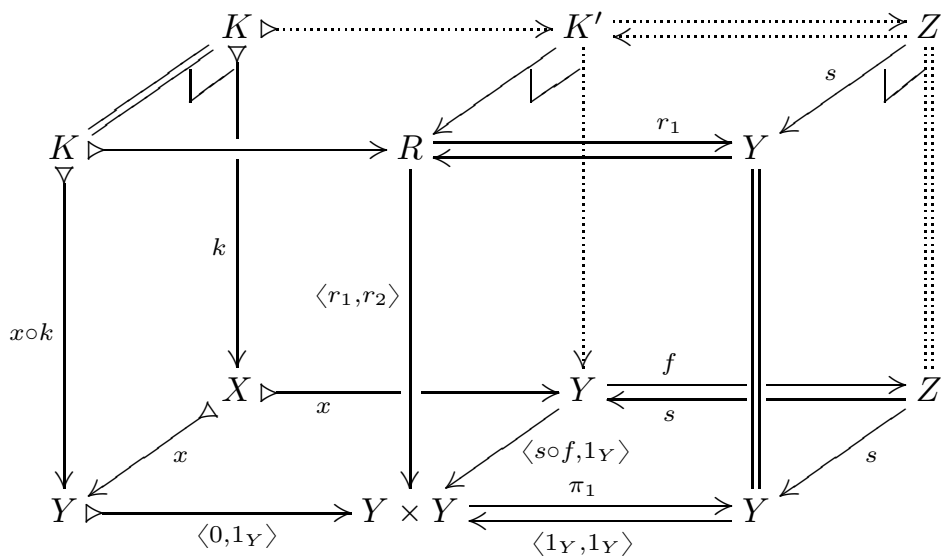

where $R$ is the denormalisation [6, 2] of $x \circ k$.

Definition 2.7. 4] A morphism $K \bowtie X$ is called a protosplit (normal) monomorphism if and only if it is the kernel of a split epimorphism. We will use the notation $K \triangleleft X$ to indicate that $K \leqslant X$ is a protosplit normal subobject of $X$, i.e. its representing monomorphisms are protosplit normal.

In what follows we shall consider the diagram

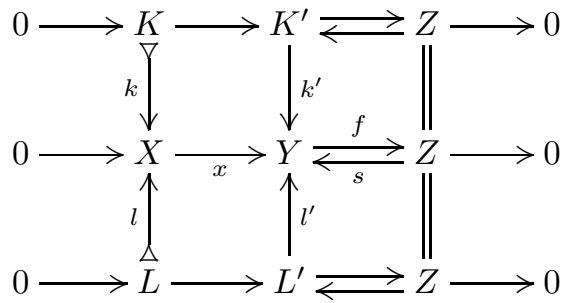

where $k^{\prime}$ and $l^{\prime}$ are normal monomorphisms in $\operatorname{Pt}_{Z}(\mathscr{C})$.

Theorem 2.8. For a semi-abelian category $\mathscr{C}$, the following are equivalent: 
(i) $\mathscr{C}$ satisfies $(\mathrm{NH})$;

(ii) for all $K, L \leqslant X \leqslant Y$, if $K, L \triangleleft Y$, then $[K, L]_{X}=[K, L]_{Y}$;

(iii) for all $K, L \leqslant X \triangleleft Y$, if $K, L \triangleleft Y$, then $[K, L]_{X}=[K, L]_{Y}$;

(iv) for all $K, L \leqslant X \triangleleft Y$, if $K, L \triangleleft Y$ then $[K, L]_{X}=[K, L]_{Y}$;

(v) for all $K, L \leqslant X \triangleleft Y$, if $K, L \triangleleft Y$ and $X=K \vee L$, then $[K, L]_{X}=[K, L]_{Y}$;

(vi) for all $K, L \leqslant X \triangleleft Y$, if $K, L \triangleleft Y$ and $X=K \vee L$, then $[K, L]_{X}=[K, L]_{Y}$;

(vii) for any diagram (†) there exists a normal subobject $N \triangleleft Y$ in $\operatorname{Pt}_{Z}(\mathscr{C})$ such that $\operatorname{Ker}(N \triangleleft Y)=[K, L]_{X} \triangleleft X$;

(viii) for any diagram (†) such that $X=K \vee L$, there exists a normal subobject $N \triangleleft Y$ in $\operatorname{Pt}_{Z}(\mathscr{C})$ such that $\operatorname{Ker}(N \triangleleft Y)=[K, L]_{X} \triangleleft X$.

Proof. By Lemma 2.4 (i) implies (ii). It is also clear that (ii) implies (iii), (iii) implies (iv), (iii) implies (v) and (v) implies (vi). Assuming now that (v) holds, since $[K, L]_{K \vee L}=[K, L]$ as explained above, (i) follows from the fact that since $K \vee L \longmapsto Y$ is the join of two normal monomorphisms it is normal [1, 22].

By Lemma 2.5, condition (vi) implies (v) as follows. Assuming that $K$ and $L$ are normal in $Y$, the lemma gives us $K, L \triangleleft\left(Y \times_{Y / X} Y\right)$. Hence by the assumption (vi) and the fact that $\langle x, 0\rangle: X \rightarrow Y \times_{Y / X} Y$ is a protosplit normal monomorphism, we

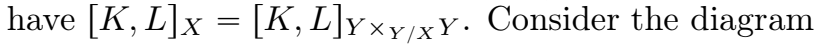

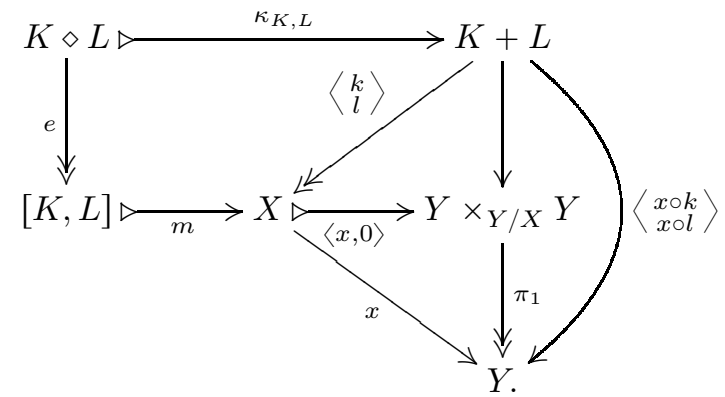

Since $X=K \vee L$ it follows that $[K, L]_{X}=[K, L]$ and so $[K, L]=[K, L]_{Y \times_{Y / X} Y}$ is normal in $Y \times_{Y / X} Y$. Since the image of a normal monomorphism is normal it follows that $x \circ m$ is normal in $Y$. Therefore since $[K, L]_{Y} \triangleleft Y$ is the normal closure of $[K, L] \leqslant Y$ it follows that $[K, L]_{Y}=[K, L]=[K, L]_{X}$ as required. Finally we note that (vii) is equivalent to (iv) and (viii) is equivalent to (vi) since they are simple reformulations obtained using Lemma 2.6] and the fact that for a morphism of split extensions

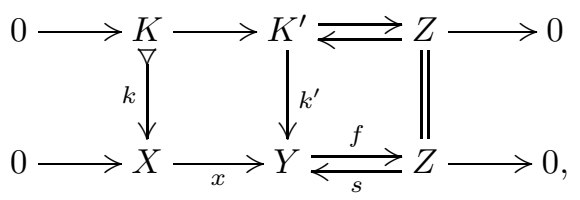

the monomorphism $k^{\prime}$ considered as a morphism in $\operatorname{Pt}_{Z}(\mathscr{C})$ is normal if and only if $x \circ k$ is normal in $\mathscr{C}$.

\section{Characteristic subobjects and (NH)}

In the category of groups it is well known that for each group $G$ the commutator $[G, G]$ is a characteristic subgroup of $G$. It is not difficult to see that a subgroup $S$ of $G$ is characteristic if and only if for each group $B$, every action $B b G \rightarrow G$, defined with respect to the monad $B b(-)$ (see [3, 4] and [1]), restricts to an action $B b S \rightarrow S$. This description was used by the first author and A. Montoli in [16] as a definition of characteristic subobject in an arbitrary semi-abelian category. In this section we will give alternative characterisations of characteristic subobjects 
and then show that (NH) implies that when $K$ and $L$ are characteristic subobjects of $X$ the Huq commutator of $[K, L]_{X}$ is a characteristic subobject of $X$. The same result was proved in [16] in a context including categories of interest.

Definition 3.1. 16] A subobject $S \leqslant X$ is said to be characteristic when every action $B b X \rightarrow X$ restricts to an action $B b S \rightarrow S$.

Proposition 3.2. For a subobject $S \leqslant X$ the following are equivalent:

(i) $S$ is a characteristic subobject of $X$;

(ii) each split extension

$$
X \stackrel{x}{\longrightarrow} Y \stackrel{f}{\stackrel{s}{\rightleftarrows}} Z
$$

lifts to a morphism of split extensions

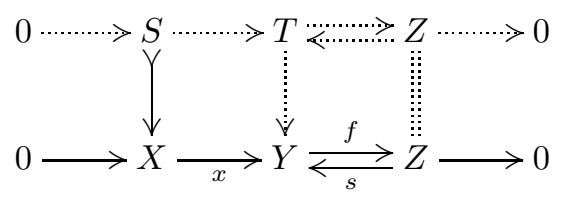

(iii) for each $Y$ such that $X \triangleleft Y, S$ is a normal subobject of $Y$;

(iv) for each $Y$ such that $X \triangleleft Y, S$ is a normal subobject of $Y$.

Proof. The implications (i) $\Leftrightarrow$ (ii) and (i) $\Rightarrow$ (iv) were proved in $[16$. It follows that the proof will be complete if we show that (iv) $\Rightarrow$ (iii) $\Rightarrow$ (ii). Trivially (iv) $\Rightarrow$ (iii) since (iii) is a special case of (iv). Finally, the implication (iii) $\Rightarrow$ (ii) follows from Lemma 2.6.

Proposition 3.3. If $\mathscr{C}$ satisfies $(\mathrm{NH})$, then for $K$ and $L$ characteristic subobjects of $X,[K, L]_{X}$ is a characteristic subobject of $X$.

Proof. By Proposition 3.2 it is sufficient to show that if $X$ is a normal subobject of $Y$ then $[K, L]_{X}$ is a normal subobject of $Y$. But, trivially by Proposition 3.2 we have $K$ and $L$ are normal subobjects of $Y$ and so by Theorem 2.8 , $[K, L]_{X}=[K, L]_{Y}$ is a normal subobject of $Y$.

Corollary 3.4. If $\mathscr{C}$ satisfies $(\mathrm{NH})$, then for each object $X$, the commutator $[X, X]=[X, X]_{X}$ is a characteristic subobject of $X$.

Proof. This follows from Proposition 3.3 with $K=L=X$, since $X$ is trivially a characteristic subobject of $X$.

\section{Categories of interest Satisfy (NH)}

The condition (NH) was first studied in the first author's thesis [15, where it is also shown that any category of interest in the sense of Orzech satisfies it. In this section we recall this fact.

Definition 4.1. 31] A category of interest is a variety of universal algebras whose theory contains a unique constant 0 , a set $\Omega$ of finitary operations and a set of identities $\mathbb{E}$ such that:

(COI 1) $\Omega=\Omega_{0} \cup \Omega_{1} \cup \Omega_{2}$, where $\Omega_{i}$ is the set of $i$-ary operations;

(COI 2) $\Omega_{0}=\{0\},-\in \Omega_{1}$ and $+\in \Omega_{2}$, where $\Omega_{i}$ is the set of $i$-ary operations, and $\mathbb{E}$ includes the group laws for $0,-,+$; define $\Omega_{1}^{\prime}=\Omega_{1} \backslash\{-\}, \Omega_{2}^{\prime}=\Omega_{2} \backslash\{+\}$;

(COI 3) for any $* \in \Omega_{2}^{\prime}$, the set $\Omega_{2}^{\prime}$ contains $\bullet$ defined by $x \bullet y=y * x$;

(COI 4) for any $\omega \in \Omega_{1}^{\prime}$, $\mathbb{E}$ includes the identity $\omega(x+y)=\omega(x)+\omega(y)$;

(COI 5) for any $* \in \Omega_{2}^{\prime}$, $\mathbb{E}$ includes the identity $x *(y+z)=x * y+x * z$;

(COI 6) for any $\omega \in \Omega_{1}^{\prime}$ and $* \in \Omega_{2}^{\prime}, \mathbb{E}$ includes the identity $\omega(x) * y=\omega(x * y)$; 
(COI 7) for any $* \in \Omega_{2}^{\prime}, \mathbb{E}$ includes the identity $x+(y * z)=(y * z)+x$;

$(\mathrm{COI} 8)$ for any $*, \cdot \in \Omega_{2}^{\prime}$, there exists a word $w$ such that $\mathbb{E}$ includes the identity

$(x * y) \cdot z=w\left(x *_{1}\left(y \mathbf{\bullet}_{1} z\right), \ldots, x *_{m}\left(y \mathbf{\bullet}_{m} z\right), y *_{m+1}\left(x \mathbf{\bullet}_{m+1} z\right), \ldots, y *_{n}\left(x \mathbf{\bullet}_{n} z\right)\right)$ where $*_{1}, \ldots, *_{n}$ and $\mathbf{-}_{1}, \ldots, \mathbf{\bullet}_{n}$ are operations in $\Omega_{2}^{\prime}$

Note that we have slightly generalized the original axiom (COI8). Note also that since any category of interest is a variety of $\Omega$-groups, it is automatically semi-abelian [24].

Examples 4.2. The categories Gp of groups, Rng of non-unitary rings, and $\mathbb{K}$-Lie of Lie algebras, $\mathbb{K}$-Leibniz of Leibniz algebras, and $\mathbb{K}$-Poisson of Poisson algebras over a field $\mathbb{K}$ are all categories of interest. On the other hand, categories of Jordan algebras and of non-associative algebras aren't, because (COI 8) fails for them. More generally, varieties of distributive $\Omega$-groups [21] need not be categories of interest.

Remark 4.3. By (COI5), for any $* \in \Omega_{2}^{\prime}$, $\mathbb{E}$ includes the identity

$$
\begin{aligned}
(x * z)+(x * t)+(y * z) & +(y * t) \\
= & (x+y) *(z+t)=(x * z)+(y * z)+(x * t)+(y * t)
\end{aligned}
$$

hence it also includes $(x * t)+(y * z)=(y * z)+(x * t)$.

Theorem 4.4. [15, Theorem 5.3.6] If $\mathscr{C}$ is a category of interest, $X$ an object in $\mathscr{C}$ and $K, L \triangleleft X$, then the Higgins commutator $[K, L]$ is normal in $X$.

Proof. Since categories of interest are distributive $\Omega$-groups by definition, according to Theorem $4 \mathrm{~B}$ in $[21$, the commutator $[K, L]$ is the ideal of $K \vee L$ generated by the elementary commutator words $\bar{w}(\mathbf{k}, \mathbf{l})=-w(\mathbf{k})-w(\mathbf{l})+w(\mathbf{k}+\mathbf{l})$, with $\mathbf{k} \in K^{n}$, $\mathbf{l} \in L^{n}$ and $w$ a single $n$-ary operation (for any $n$ ) or the identity. Since $\mathscr{C}$ is a category of interest, we have elementary commutator words of these types:

(i) $-k-l+k+l$, with $k \in K$ and $l \in L$;

(ii) $-\omega(k)-\omega(l)+\omega(k+l)$, with $k \in K, l \in L$ and $\omega \in \Omega_{1}^{\prime}$;

(iii) $-\left(k_{1} * k_{2}\right)-\left(l_{1} * l_{2}\right)+\left(\left(k_{1}+l_{1}\right) *\left(k_{2}+l_{2}\right)\right)$, with $k_{1}, k_{2} \in K, l_{1}, l_{2} \in L$ and $* \in \Omega_{2}^{\prime}$.

Now, by Definition 4.1

$$
-\omega(k)-\omega(l)+\omega(k+l) \stackrel{(\mathrm{COI} 4)}{=}-\omega(k)-\omega(l)+\omega(k)+\omega(l)
$$

and $\omega(k) \in K, \omega(l) \in L$, since $K$ and $L$ are subobjects. It follows that words of type (ii) are again of type (i). Moreover,

$$
\begin{aligned}
-\left(k_{1} * k_{2}\right)-\left(l_{1} * l_{2}\right)+\left(\left(k_{1}+l_{1}\right) *\left(k_{2}+l_{2}\right)\right) & \\
\left(\stackrel{\mathrm{COI} 5)}{=}-\left(k_{1} * k_{2}\right)-\left(l_{1} * l_{2}\right)+\left(k_{1} * k_{2}\right)+\left(k_{1} * l_{2}\right)+\right. & \left(l_{1} * k_{2}\right)+\left(l_{1} * l_{2}\right) \\
& =\left(k_{1} * l_{2}\right)+\left(l_{1} * k_{2}\right),
\end{aligned}
$$

where the last equality follows from Remark 4.3.

So we only have two types of elementary commutator words:

(i) $-k-l+k+l$, with $k \in K$ and $l \in L$;

(iii)' $\left(k_{1} * l_{2}\right)+\left(l_{1} * k_{2}\right)$, with $k_{1}, k_{2} \in K, l_{1}, l_{2} \in L$ and $* \in \Omega_{2}^{\prime}$.

Now let $\llbracket K, L \rrbracket$ be the subobject of $X$ generated by the elementary commutator words. We shall prove that $\llbracket K, L \rrbracket$ is normal in $X$ (and thus in $K \vee L$ ). As a consequence, $\llbracket K, L \rrbracket=[K, L]$ and $[K, L]$ will be an ideal of $X$, as required.

In order to prove that $\llbracket K, L \rrbracket$ is an ideal of $X$, by Theorem 1.7 in [31] it suffices to prove that it is closed under conjugation and products with elements of $X$. We start by verifying these two conditions for generators. 
(i) For any $k \in K, l \in L, x \in X$ :

$$
\begin{aligned}
-x+(-k-l+ & k+l)+x \\
& =(-x-k+x)+(-x-l+x)+(-x+k+x)+(-x+l+x)
\end{aligned}
$$

and the right hand expression is an elementary commutator word of type (i), $K$ and $L$ being ideals of $X$; for any $k \in K, l \in L, x \in X$ and $* \in \Omega_{2}^{\prime}$ :

$$
(-k-l+k+l) * x \stackrel{(\text { COI } 5)}{=}-(k * x)-(l * x)+(k * x)+(l * x)
$$

and again the right hand expression is an elementary commutator word of type (i), $K$ and $L$ being ideals of $X$.

(iii)' For any $k_{1}, k_{2} \in K, l_{1}, l_{2} \in L, x \in X$ and $* \in \Omega_{2}^{\prime}$

$$
-x+\left(k_{1} * l_{2}\right)+\left(l_{1} * k_{2}\right)+x \stackrel{(\mathrm{COI} 7)}{=}\left(k_{1} * l_{2}\right)+\left(l_{1} * k_{2}\right),
$$

that is, commutator words of type (iii)' are stable by conjugation; for any $k_{1}$, $k_{2} \in K, l_{1}, l_{2} \in L, x \in X$ and $*, \cdot \in \Omega_{2}^{\prime}$,

$$
\left(\left(k_{1} * l_{2}\right)+\left(l_{1} * k_{2}\right)\right) \cdot x \stackrel{(\text { COI } 5)}{=}\left(\left(k_{1} * l_{2}\right) \cdot x\right)+\left(\left(l_{1} * k_{2}\right) \cdot x\right)
$$

but, by (COI 8$)$, there exists a word $w$ such that

$$
\begin{aligned}
& \left(k_{1} * l_{2}\right) \cdot x= \\
& w\left(k_{1} *_{1}\left(l_{2} \mathbf{\bullet}_{1} x\right), \ldots, k_{1} *_{m}\left(l_{2} \mathbf{\bullet}_{m} x\right), l_{2} *_{m+1}\left(k_{1} \mathbf{\bullet}_{m+1} x\right), \ldots, l_{2} *_{n}\left(k_{1} \mathbf{\bullet}_{n} x\right)\right)
\end{aligned}
$$

where each term on the right is an elementary commutator word since $K$ and $L$ are ideals, and so the product $\left(k_{1} * l_{2}\right) \boldsymbol{\bullet} x$ is generated by elementary commutator words. Similarly, the same holds for the product $\left(l_{1} * k_{2}\right) \cdot x$.

We conclude the proof by induction. Let $w_{1}(\mathbf{k}, \mathbf{l})$ and $w_{2}\left(\mathbf{k}^{\prime}, \mathbf{l}^{\prime}\right)$ be words in $\llbracket K, L \rrbracket$ satisfying the two conditions above - conjugates and products with elements of $X$ are still in $\llbracket K, L \rrbracket$. Let us first consider the sum $w_{1}+w_{2}$. For any $x \in X$,

$$
-x+\left(w_{1}+w_{2}\right)+x=\left(-x+w_{1}+x\right)+\left(-x+w_{2}+x\right)
$$

and the right hand expression is in $\llbracket K, L \rrbracket$, since $w_{1}$ and $w_{2}$ satisfy the induction hypothesis. For any $x \in X$ and $* \in \Omega_{2}^{\prime}$,

$$
\left(w_{1}+w_{2}\right) * x \stackrel{(\mathrm{COI} 5)}{=}\left(w_{1} * x\right)+\left(w_{2} * x\right)
$$

and again the right hand expression is in $\llbracket K, L \rrbracket$, since $w_{1}$ and $w_{2}$ satisfy the induction hypothesis.

Now consider the product $w_{1} * w_{2}$ where $*$ is a fixed operation in $\Omega_{2}^{\prime}$. For any $x \in X$,

$$
-x+\left(w_{1} * w_{2}\right)+x \stackrel{(\mathrm{COI} 7)}{=} w_{1} * w_{2},
$$

that is, the product $w_{1} * w_{2}$ is stable under conjugation; and for any $x \in X$ and - $\in \Omega_{2}^{\prime}$,

$$
\begin{aligned}
\left(w_{1} * w_{2}\right) \cdot x \stackrel{(\mathrm{COI} 8)}{=} w\left(w_{1} *_{1}\left(w_{2} \bullet_{1} x\right), \ldots, w_{1} *_{m}\left(w_{2} \bullet_{m} x\right)\right. \\
\left.w_{2} *_{m+1}\left(w_{1} \bullet_{m+1} x\right), \ldots, w_{2} *_{n}\left(w_{1} \bullet_{n} x\right)\right)
\end{aligned}
$$

where each term on the right is generated by elementary commutator words, because $w_{1}$ and $w_{2}$ satisfy the induction hypothesis. Hence $\left(w_{1} * w_{2}\right) \cdot x \in \llbracket K, L \rrbracket$, which concludes the proof.

Next we give an example showing that $(\mathrm{NH})$ need not hold outside the context of categories of interest. In particular (COI 7) is necessary. Example 5.4 below proves that also (COI 8 ) is also necessary. Both show that there are semi-abelian categories in which Higgins commutators of normal subobjects need not be normal. 
Example 4.5. [15, Example 5.3.8] Consider the category whose objects are groups with an additional binary associative and distributive operation $*$, which satisfies all the axioms in Definition 4.1. except for (COI7), and morphisms are group homomorphisms preserving $*$. Let $A$ be the dihedral group of order 8 , in additive notation:

$$
A=\langle r, s \mid 4 r=0,2 s=0, s+r+s=3 r\rangle
$$

endowed with an associative and distributive product generated by:

\begin{tabular}{l|ll}
$*$ & $r$ & $s$ \\
\hline$r$ & $s$ & $s$ \\
$s$ & $s$ & $s$
\end{tabular}

The subobject $K=\{0,2 r, s, s+2 r\}$ of $A$ generated by $s$ and $2 r$ is an ideal of $A$, whereas the commutator $[K, K]=\{0, s\}$ is not, since it is not closed under conjugation: indeed, $r+s-r=r+s+3 r=s+2 r \notin[K, K]$.

\section{Independence of The Smith is Huq COndition (SH)}

In this section we prove that $(\mathrm{NH})$ is independent of the Smith is Huq condition $(\mathrm{SH})$, by giving examples of categories which satisfy one but not the other.

5.1. The Smith is $\boldsymbol{H u q}$ condition (SH). Given two equivalence relations $R$ and $S$ on $X$, with respective normalisations $K, L \triangleleft X$, the Smith commutator $[R, S]^{\mathrm{S}}$ of $R$ and $S$ is an equivalence relation on $X$ which measures how far $R$ and $S$ are from centralising each other (see [34, 32, 2]). If the Smith commutator of two equivalence relations is trivial, then the Huq commutator of their normalisations is also trivial [10]. But, in general, the converse is false; in [2, 8] a counterexample is given in the category of digroups, which is a semi-abelian variety, even a variety of $\Omega$-groups [21]. The requirement that the two commutators vanish together is known as the Smith is Huq Condition (SH) and it is shown in 28 that, for a semi-abelian category, this condition holds if and only if every star-multiplicative graph is an internal groupoid, which is important in the study of internal crossed modules 23. Moreover, $(\mathrm{SH})$ is also known to hold for pointed strongly protomodular categories [10] (in particular, for any Moore category [17, 33]) and in action accessible categories [12] (in particular, for any category of interest [30]).

5.2. Characterisation in terms of Higgins commutators. Given subobjects $k: K \rightarrow X, l: L \rightarrow X$ and $m: M \rightarrow X$ of an object $X$, the ternary Higgins commutator $[K, L, M] \leqslant X$ is the image of the composite

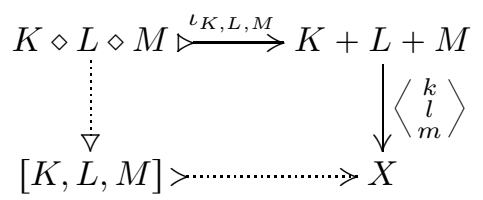

where $\iota_{K, L, M}$ is the kernel of

$$
K+L+M \stackrel{\left\langle\begin{array}{ccc}
i_{K} & i_{K} & 0 \\
i_{L} & 0 & i_{L} \\
0 & i_{M} & i_{M}
\end{array}\right\rangle}{\longrightarrow}(K+L) \times(K+M) \times(L+M) ;
$$

$i_{k}, i_{L}$ and $i_{M}$ denote coproduct injection morphisms. The object $K \diamond L \diamond M$ is the ternary co-smash product [14, 20, 19] of $K, L$ and $M$.

The main result of [20] states that for all $K, L \triangleleft X$, the Smith commutator $[K, L]^{\mathrm{S}}$ may be decomposed as the join $[K, L] \vee[K, L, X]$, so that $(\mathrm{SH})$ holds if and only if $[K, L]_{X}=[K, L] \vee[K, L, X]$ or, equivalently, $[K, L, X] \leqslant[K, L]_{X}$. 
5.3. Relation between $(\mathrm{NH})$ and $(\mathrm{SH})$. It is a natural question to ask whether the conditions $(\mathrm{NH})$ and $(\mathrm{SH})$ are related. The following examples show that they are, in fact, independent.

Example 5.4. [15, Example 5.3.7] Let NARng be the category non-associative rings [21] whose objects are abelian groups with an additional binary operation * which distributes over addition; and whose morphisms are group homomorphisms preserving *. This category satisfies all axioms in Definition 4.1, except for (COI 8).

Let $A$ be the object in NARng with abelian group structure the free abelian group on $\{x, y, z\}$, endowed with a distributive product with the following multiplication table:

\begin{tabular}{c|ccc}
$*$ & $x$ & $y$ & $z$ \\
\hline$x$ & $x$ & 0 & $y$ \\
$y$ & 0 & 0 & $x$ \\
$z$ & $y$ & $x$ & $z$
\end{tabular}

The subobject $K$ generated by $x$ and $y$ is an ideal of $A$, whereas the commutator $[K, K]$, which is the subobject generated by $x$ is not, because it is not closed under multiplication with external elements: $x * z=y \notin[K, K]$.

Since the category NARng is strongly protomodular (being a variety of distributive $\Omega_{2}$-groups [26]), it follows that strong protomodularity [5, 8] does not imply $(\mathrm{NH})$. In particular, since a strongly protomodular semi-abelian category always satisfies $(\mathrm{SH})$ it follows that a semi-abelian category may satisfy ( $\mathrm{SH}$ ) but $\operatorname{not}(\mathrm{NH})$.

Example 5.5. Let $\mathscr{C}$ be the category whose objects are abelian groups endowed with a symmetric and distributive ternary operation $t$ satisfying the following associativity property:

$$
t(t(x, y, z), u, v)=t(t(x, u, v), y, z)
$$

Morphisms are as usual maps preserving all operations. Since $\mathscr{C}$ is a variety of distributive $\Omega$-groups, we know from [21, Theorems $4 \mathrm{~A}, 4 \mathrm{C}$ ] that, given $K, L \leqslant X$ in $\mathscr{C}$ :

(i) $K \triangleleft X$ if and only if for all $k \in K$ and $x_{1}, x_{2} \in X$ also $t\left(k, x_{1}, x_{2}\right) \in K$;

(ii) $[K, L]$ is generated by elements of type $t\left(k_{1}, k_{2}, l_{2}\right)$ or $t\left(k_{1}, l_{1}, l_{2}\right)$, where $k_{1}, k_{2} \in K$ and $l_{1}, l_{2} \in L$.

For $K \leqslant X$, if $k_{1}, k_{2}, k_{3}$ are elements of $K$ and $x_{1}, x_{2}$ are elements of $X$ such that $t\left(k_{i}, x_{1}, x_{2}\right) \in K$, then

$$
\begin{gathered}
t\left(k_{1}+k_{2}, x_{1}, x_{2}\right)=t\left(k_{1}, x_{1}, x_{2}\right)+t\left(k_{2}, x_{1}, x_{2}\right) \in K \\
t\left(t\left(k_{1}, k_{2}, k_{2}\right), x_{1}, x_{2}\right)=t\left(t\left(k_{1}, x_{1}, x_{2}\right), k_{2}, k_{3}\right) \in K .
\end{gathered}
$$

Hence it is sufficient to check (i) on generators. As a consequence, if $K, L \triangleleft X$ then $[K, L]$ is normal in $X$, since

$$
t\left(t\left(k_{1}, k_{2}, l_{2}\right), u, v\right)=t\left(t\left(k_{1}, u, v\right), k_{2}, l_{2}\right) \in[K, L]
$$

for all $u, v \in X$, and a similar argument holds for the terms of second type. This shows that $(\mathrm{NH})$ holds in $\mathscr{C}$.

Consider now the object of $\mathscr{C}$ consisting of the abelian group $\mathbb{Z}$ with the operation $t(x, y, z)=x y z$. Then, if we consider the subobjects $2 \mathbb{Z}$ and $4 \mathbb{Z}$, it happens that $[2 \mathbb{Z}, 4 \mathbb{Z}]=16 \mathbb{Z}$, while $[2 \mathbb{Z}, 4 \mathbb{Z}, \mathbb{Z}]=8 \mathbb{Z}$. So $[2 \mathbb{Z}, 4 \mathbb{Z}]^{\mathrm{S}}=[2 \mathbb{Z}, 4 \mathbb{Z}] \vee[2 \mathbb{Z}, 4 \mathbb{Z}, \mathbb{Z}]>$ $[2 \mathbb{Z}, 4 \mathbb{Z}]$ showing that $\mathscr{C}$ does not satisfy $(\mathrm{SH})$. 


\section{Equivalent characterisations of $(\mathrm{SH})+(\mathrm{NH})$}

Many categories - all categories of interest, for instance, as explained in 4.4 and 5.1 do actually satisfy both $(\mathrm{SH})$ and $(\mathrm{NH})$. These two conditions, when required together, may be characterised in terms of the fibration of points as shown in Theorem6.5. We begin with a straightforward characterisation in terms of ternary commutators.

Proposition 6.1. A semi-abelian category $\mathscr{C}$ satisfies $(\mathrm{SH})+(\mathrm{NH})$ if and only if for all $K, L \triangleleft X$ in $\mathscr{C}$,

$$
[K, L, X] \leqslant[K, L] .
$$

Proof. This follows from the chain of inclusions

$$
[K, L] \leqslant[K, L]_{X} \leqslant[K, L] \vee[K, L, X]
$$

and the fact that the Smith commutator of the equivalence relations corresponding to $K$ and $L$ has the join on the right as its normalisation [20].

This immediately implies that any semi-abelian category $\mathscr{C}$ which satisfies $(\mathrm{SH})+(\mathrm{NH})$ is peri-abelian in the sense of [9, since, via the characterisation in [18, $\mathscr{C}$ is such if and only if for all $K \triangleleft X$ we have $[K, K, X] \leqslant[K, K]$.

It was proved in [13, 29] that $(\mathrm{SH})$ is equivalent to the condition that kernel functors reflect Huq commutativity of normal subobjects. By (vii) in Theorem 2.8, condition $(\mathrm{NH})$ is equivalent to the condition that Huq commutators of cospans of normal monomorphisms which are the image of cospans of normal monomorphisms under a kernel functor are themselves images of normals subobjects under the same kernel functor. Hence we are able to study these properties together using an abstract functor as in Lemmas 6.3 and 6.4 below, and make conclusions about the condition $(\mathrm{NH})+(\mathrm{SH})$ in Theorem 6.5

Definition 6.2. A class $\mathbb{C}$ of cospans in $\mathscr{C}$ is closed under (direct) images when for any cospan $(k: K \rightarrow X, l: L \rightarrow X)$ in $\mathbb{C}$ and any regular epimorphism $e: X \rightarrow X^{\prime}$ in $\mathscr{C}$, the cospan $\left(k^{\prime}, l^{\prime}\right)$ where $k^{\prime}$ and $l^{\prime}$ are the images of $e \circ k$ and $e \circ l$, respectively, is in $\mathbb{C}$.

Recall that a functor is said to be conservative when it reflects isomorphisms.

Lemma 6.3. Let $\mathscr{C}$ and $\mathscr{D}$ be semi-abelian categories, let $\mathbb{C}$ be a class of cospans in $\mathscr{C}$ which is closed under images, let $F: \mathscr{C} \rightarrow \mathscr{D}$ be a conservative functor which preserves limits and regular epimorphisms, and let $\mathbb{D}$ be the image of $\mathbb{C}$ under $F$. The following are equivalent:

(i) F reflects Huq commutativity of those cospans in $\mathbb{D}$;

(ii) F reflects Huq commutators of those cospans in $\mathbb{D}$.

Proof. The implication (ii) $\Rightarrow$ (i) follows from the fact that the functor $F$ preserves the zero object. To prove (i) $\Rightarrow$ (ii), let $(k: K \rightarrow X, l: L \rightarrow X)$ be a cospan in $\mathbb{C}$ and suppose there exists a normal monomorphism $w: W \rightarrow X$ such that $F(w)$ is the Huq commutator of $F(k)$ and $F(l)$. Let $e$ be the cokernel of $w$ as displayed in the the short exact sequence

$$
0 \longrightarrow W \stackrel{w}{\longrightarrow} X \stackrel{e}{\longrightarrow} X / W \longrightarrow 0
$$

and let $k^{\prime}$ and $l^{\prime}$ be the images of $e \circ k$ and $e \circ l$. Since $F$ preserves limits and regular epimorphisms it preserves short exact sequences (since regular epimorphisms are normal in $\mathscr{D})$, and so $F(e)$ is the quotient of the Huq commutator of $F(k)$ and $F(l)$, which by definition means that $F(e \circ k)$ and $F(e \circ l)$ and so by [2] that $F\left(k^{\prime}\right)$ and $F\left(l^{\prime}\right)$ commute. Since $\mathbb{C}$ is closed under images, $\left(k^{\prime}, l^{\prime}\right)$ is in $\mathbb{C}$ and so, by (i), $k^{\prime}$ and $l^{\prime}$ and therefore $e \circ k$ and $e \circ l$ commute. It follows that $[K, L]_{X} \leqslant W$ and 
therefore that $F\left([K, L]_{X}\right) \leqslant F(W)$. Since $F$ preserves Huq commutativity (since it preserves limits) and short exact sequences it follows that

$$
F(W)=[F(K), F(L)]_{F(X)} \leqslant F\left([K, L]_{X}\right),
$$

meaning that $F\left([K, L]_{X}\right)=F(W)$, and therefore since $F$ reflects isomorphisms $[K, L]_{X}=W$ as required.

Lemma 6.4. Let $\mathscr{C}$ and $\mathscr{D}$ be semi-abelian categories, let $\mathbb{C}$ be a class of cospans in $\mathscr{C}$ which is closed under images, let $F: \mathscr{C} \rightarrow \mathscr{D}$ be a conservative functor which preserves limits and regular epimorphisms, and let $\mathbb{D}$ be the image of $\mathbb{C}$ under $F$. The following are equivalent:

(i) F reflects Huq commutativity of cospans in $\mathbb{D}$, and Huq commutators of cospans in $\mathbb{D}$ are the image of normal subobjects under $F$;

(ii) F reflects Huq commutators of cospans in $\mathbb{D}$, and Huq commutators of cospans in $\mathbb{D}$ are the image of normal subobjects under $F$;

(iii) F preserves Huq commutators of cospans in $\mathbb{C}$.

Proof. The equivalence of (i) and (ii) follows from Lemma 6.3. Since $F$ reflects isomorphisms, it easily follows that (iii) $\Rightarrow$ (ii). To prove that (ii) $\Rightarrow$ (iii), let $(k: K \rightarrow X, l: L \rightarrow X)$ be a cospan in $\mathbb{C}$. By the second part of (ii), there exists a normal monomorphism $w: W \rightarrow X$ such that $F(w)$ is the Huq commutator of $F(k)$ and $F(l)$. It follows that since $F$ reflects such commutators, $w$ is the commutator of $k$ and $l$ meaning that $F$ preserves commutators of cospans in $\mathbb{C}$ as required.

Now we apply this to the situation where $F=\operatorname{Ker}: \operatorname{Pt}_{Z}(\mathscr{C}) \rightarrow \mathscr{C}$ is a kernel functor and $\mathbb{C}$ is the class of cospans of normal monomorphisms.

Theorem 6.5. For a semi-abelian category $\mathscr{C}$, the following are equivalent:

(i) $\mathscr{C}$ satisfies $(\mathrm{SH})+(\mathrm{NH})$;

(ii) $\mathscr{C}$ satisfies $(\mathrm{NH})$ and the kernel functors $\mathrm{Ker}: \mathrm{Pt}_{Z}(\mathscr{C}) \rightarrow \mathscr{C}$ reflect Huq commutators of pairs of normal subobjects;

(iii) the kernel functors $\mathrm{Ker}: \mathrm{Pt}_{Z}(\mathscr{C}) \rightarrow \mathscr{C}$ preserve Huq commutators of pairs of normal subobjects;

(iv) $\mathscr{C}$ satisfies $(\mathrm{NH})$ and the change of base functors $f^{*}: \operatorname{Pt}_{Z}(\mathscr{C}) \rightarrow \operatorname{Pt}_{W}(\mathscr{C})$ of the fibration of points reflect Huq commutators of pairs of normal subobjects;

(v) the change of base functors $f^{*}: \mathrm{Pt}_{Z}(\mathscr{C}) \rightarrow \mathrm{Pt}_{W}(\mathscr{C})$ of the fibration of points preserve Huq commutators of pairs of normal subobjects;

(vi) for each $Z$ in $\mathscr{C}$ the category $\mathrm{Pt}_{Z}(\mathscr{C})$ satisfies $(\mathrm{SH})+(\mathrm{NH})$.

Proof. As explained above, (i) is equivalent to (ii). The equivalence between (ii) and (iii) follows from Lemma 6.4. Next we will show that (ii) + (iii) implies (iv) $+($ v). Let $f: W \rightarrow Z$ be a morphism $\mathscr{C}$. Consider the diagram of induced pullback functors

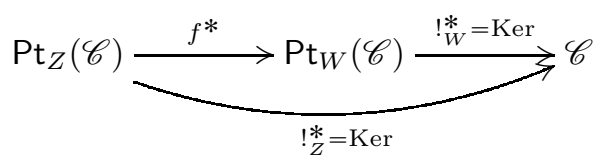

which commutes (up to natural isomorphism). It is clear that $f^{*}$ preserves Huq commutators of pairs of normal subobjects because the kernel functor $!_{Z}^{*}$ preserves them and $!_{W}^{*}$ reflects them. On the other hand, $f^{*}$ reflects Huq commutators of pairs of normal subobjects because $!_{W}^{*}$ preserves them and $!_{Z}^{*}$ reflects them. The 
implications (vi) $\Rightarrow(\mathrm{i})$, (v) $\Rightarrow$ (iii) and (iv) $\Rightarrow$ (ii) are obvious. Finally, since there is an isomorphism of categories $\mathrm{Pt}_{(A, p, s)}\left(\mathrm{Pt}_{B}(\mathscr{C})\right) \cong \mathrm{Pt}_{A}(\mathscr{C})$ making the diagram

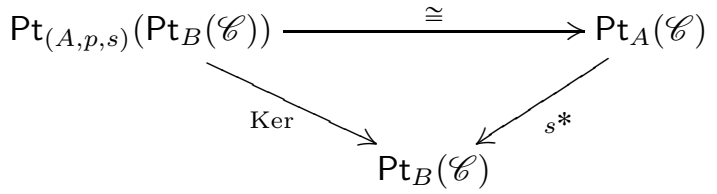

commute, it follows that (v) for $\mathscr{C}$ implies (iii) for $\operatorname{Pt}_{B}(\mathscr{C})$ which then implies (i) for $\operatorname{Pt}_{B}(\mathscr{C})$.

\section{REFERENCES}

[1] F. Borceux, A survey of semi-abelian categories, in Janelidze et al. 25], pp. 27-60.

[2] F. Borceux and D. Bourn, Mal'cev, protomodular, homological and semi-abelian categories, Math. Appl., vol. 566, Kluwer Acad. Publ., 2004.

[3] F. Borceux, G. Janelidze, and G. M. Kelly, Internal object actions, Comment. Math. Univ. Carolinae 46 (2005), no. 2, 235-255.

[4] F. Borceux, G. Janelidze, and G. M. Kelly, On the representability of actions in a semiabelian category, Theory Appl. Categ. 14 (2005), no. 11, 244-286.

[5] D. Bourn, Normal functors and strong protomodularity, Theory Appl. Categ. 7 (2000), no. 9, 206-218.

[6] D. Bourn, Normal subobjects and abelian objects in protomodular categories, J. Algebra 228 (2000), 143-164.

[7] D. Bourn, Commutator theory in regular Mal'cev categories, in Janelidze et al. [25], pp. 61-76.

[8] D. Bourn, Commutator theory in strongly protomodular categories, Theory Appl. Categ. 13 (2004), no. 2, 27-40.

[9] D. Bourn, The cohomological comparison arising from the associated abelian object, preprint arXiv:1001.0905, 2010.

[10] D. Bourn and M. Gran, Centrality and normality in protomodular categories, Theory Appl. Categ. 9 (2002), no. 8, 151-165.

[11] D. Bourn and G. Janelidze, Protomodularity, descent, and semidirect products, Theory Appl. Categ. 4 (1998), no. 2, 37-46.

[12] D. Bourn and G. Janelidze, Centralizers in action accessible categories, Cah. Topol. Géom. Differ. Catég. L (2009), no. 3, 211-232.

[13] D. Bourn, N. Martins-Ferreira, and T. Van der Linden, A characterisation of the "Smith is Huq" condition in the pointed Mal'tsev setting, Cah. Topol. Géom. Différ. Catégor. LIV (2013), no. 3, 163-183.

[14] A. Carboni and G. Janelidze, Smash product of pointed objects in lextensive categories, J. Pure Appl. Algebra 183 (2003), 27-43.

[15] A. S. Cigoli, Centrality via internal actions and action accessibility via centralizers, Ph.D. thesis, Università degli Studi di Milano, 2009.

[16] A. S. Cigoli and A. Montoli, Characteristic subobjects in semi-abelian categories, PréPublicações DMUC 13-50 (2013), 1-27, submitted.

[17] M. Gerstenhaber, A categorical setting for the Baer extension theory, Applications of Categorical Algebra, New York 1968, Proc. Sympos. Pure Math., vol. XVII, Amer. Math. Soc., Providence, R.I., 1970, pp. 50-64.

[18] J. R. A. Gray and T. Van der Linden, Peri-abelian categories and the universal central extension condition, submitted, 2014.

[19] M. Hartl and B. Loiseau, On actions and strict actions in homological categories, Theory Appl. Categ. 27 (2013), no. 15, 347-392.

[20] M. Hartl and T. Van der Linden, The ternary commutator obstruction for internal crossed modules, Adv. Math. 232 (2013), no. 1, 571-607.

[21] P. J. Higgins, Groups with multiple operators, Proc. Lond. Math. Soc. (3) 6 (1956), no. 3, 366-416.

[22] S. A. Huq, Commutator, nilpotency and solvability in categories, Q. J. Math. 19 (1968), no. $2,363-389$.

[23] G. Janelidze, Internal crossed modules, Georgian Math. J. 10 (2003), no. 1, 99-114.

[24] G. Janelidze, L. Márki, and W. Tholen, Semi-abelian categories, J. Pure Appl. Algebra 168 (2002), no. 2-3, 367-386.

[25] G. Janelidze, B. Pareigis, and W. Tholen (eds.), Galois theory, Hopf algebras, and semiabelian categories, Fields Inst. Commun., vol. 43, Amer. Math. Soc., 2004. 
[26] S. Mantovani and G. Metere, Internal crossed modules and Peiffer condition, Theory Appl. Categ. 23 (2010), no. 6, 113-135.

[27] S. Mantovani and G. Metere, Normalities and commutators, J. Algebra 324 (2010), no. 9, $2568-2588$

[28] N. Martins-Ferreira and T. Van der Linden, A note on the "Smith is Huq" condition, Appl. Categ. Structures 20 (2012), no. 2, 175-187.

[29] N. Martins-Ferreira and T. Van der Linden, Further remarks on the "Smith is Huq" condition, Appl. Categ. Structures, accepted for publication, 2014

[30] A. Montoli, Action accessibility for categories of interest, Theory Appl. Categ. 23 (2010), no. $1,7-21$

[31] G. Orzech, Obstruction theory in algebraic categories I and II, J. Pure Appl. Algebra 2 (1972), 287-314 and 315-340.

[32] M. C. Pedicchio, A categorical approach to commutator theory, J. Algebra 177 (1995), 647657.

[33] D. Rodelo, Moore categories, Theory Appl. Categ. 12 (2004), no. 6, 237-247.

[34] J. D. H. Smith, Mal'cev varieties, Lecture Notes in Math., vol. 554, Springer, 1976.

E-mail address: alan.cigoli@unimi.it

E-mail address: jamesgray@sun.ac.za

E-mail address: tim.vanderlinden@uclouvain.be

Dipartimento di Matematica, Università degli Studi di Milano, Via Saldini 50, 20133 Milano, Italy

Mathematics Division, Department of Mathematical Sciences, Stellenbosch University, Private Bag X1, Matieland 7602, South Africa

Institut de Recherche en Mathématique et Physique, Université catholique de Louvain, Chemin du cyclotron 2 Bte L7.01.02, 1348 Louvain-la-Neuve, Belgium 